\title{
Composição e estrutura da comunidade de peixes de uma praia arenosa da Ilha do Frade, Vitória, Espírito Santo
}

\author{
Ciro C. V. de Araujo ${ }^{1,3}$, Daniel de M. Rosa ${ }^{1}$, Joelson M. Fernandes ${ }^{1}$, \\ Leandro V. Ripoli ${ }^{1} \&$ Werther Krohling ${ }^{2}$
}

1. Núcleo de Atividades Ambientais (NATIVA) - Av. Hugo Musso, 1333, Apto 1106, 29101-280 Vila Velha, ES. (cirovilar@nativa.org.br)

2. Centro Universitário Vila Velha - Comissário José Dantas de Melo, 21, 29102-770 Vila Velha, ES, Brasil.

3. Pós-Graduação em Ecologia e Conservação, Setor de Ciências Biológicas, Universidade Federal do Paraná, Caixa Postal 19031, 81531 980 Curitiba, PR, Brasil.

\begin{abstract}
Structure and composition of fish community from a sandy beach on Ilha do Frade, Vitória, Espírito Santo. The ichthyofauna of a sandy beach on Ilha do Frade, Vitória, Espírito Santo, was sampled monthly between May/2004 and April/2005. As many as 2,689 individuals were collected from 26 families and 45 Teleostei species, mainly juvenile forms. The Sciaenidae presented the highest number of species. The monthly collections showed that the most abundant species in number were Lutjanus synagris (Linnaeus, 1758), Archosargus rhomboidalis (Linnaeus, 1758), Eucinostomus lefroyi (Goode, 1874), and Paralonchurus brasiliensis (Steindachner, 1875); in terms of biomass, Cyclichthys spinosus (Linnaeus, 1758), A. rhomboidalis, E. lefroyi and L. synagris dominated. Number and biomass varied significantly $(\mathrm{p}<0.01)$ among months. Shannon-Wiener's Index $\left(\mathrm{H}^{\prime}\right)$ presented low monthly variation, not evidencing a seasonal tendency.
\end{abstract}

KEYWORDS. Ichthyofauna, sandy beach, community, Vitória, Espírito Santo.

RESUMO. A ictiofauna de uma praia arenosa da Ilha do Frade, Vitória, ES, foi amostrada mensalmente entre maio/2004 e abril/2005. Foram coletados 2.689 indivíduos de 26 famílias e 45 espécies de Teleostei, a maioria em estágio juvenil. A família Sciaenidae apresentou o maior número de espécies. As capturas mensais evidenciaram que as espécies numericamente mais importantes foram: Lutjanus synagris (Linnaeus, 1758), Archosargus rhomboidalis (Linnaeus, 1758), Eucinostomus lefroyi (Goode, 1874) e Paralonchurus brasiliensis (Steindachner, 1875); com relação ao peso, Cyclichthys spinosus (Linnaeus, 1758), A. rhomboidalis, E. lefroyi e L. synagris dominaram. $\mathrm{O}$ número de indivíduos e a biomassa variaram significativamente $(\mathrm{p}<0,01)$ entre os meses. O índice de Shannon-Wiener $\left(\mathrm{H}^{\prime}\right)$ apresentou pequenas variações mensais, não evidenciando uma tendência sazonal.

PALAVRAS CHAVE. Ictiofauna, praia arenosa, comunidade, Vitória, Espírito Santo.

As comunidades de peixes representam um importante elemento dentro dos ecossistemas costeiros e são relevantes como recurso pesqueiro. Devido à grande oferta de alimento e habitats existentes nas regiões costeiras, muitas das espécies de peixes marinhos escolhem estes ambientes como área de reprodução, desenvolvimento e alimentação (ModDE, 1980; RuPLE, 1984; GAELZER \& ZALMON, 2003).

Estes ambientes são caracterizados por grandes variações, tanto dos fatores abióticos quanto da composição faunística, sendo que as praias estão entre os mais dinâmicos. As praias arenosas representam o mais amplo dos ecossistemas sedimentares costeiros (DAVIS, 1985).

Considerando a extensão do litoral brasileiro de aproximadamente $9.500 \mathrm{~km}$ (KNOPPERS et al., 2002), ainda existem relativamente poucos estudos abordando a ictiofauna de praias arenosas, sendo a maioria desenvolvidos nas regiões sudeste e sul (e. g. Helmer \& Perrone, 1991; Saul \& Cunningham, 1995; Gaelzer \& Zalmon, 2003; Pessanha \& Araúuo, 2003; Godefroid $e t$ al., 1997; 2003; 2004; Felix et al., 2006) e poucos nas demais regiões (e. g. Teixeira et al., 1992; Teixeira \& AlMEIDA, 1998; LoPEs et al., 1999). Em relação ao litoral do Espírito Santo, existem poucas informações a respeito das comunidades de peixes que o utilizam (Helmer \& Perrone, 1991; Chagas et al., 2006); entretanto, mais escassos ainda são os estudos abordando a estrutura das assembléias ícticas de praias arenosas, apesar de sua freqüência no Estado.

Devido às particularidades de cada ambiente costeiro, a zona de arrebentação das praias pode fornecer diferentes oportunidades para ambientação de peixes juvenis (Clark et al., 1996; Clark, 1997).

O presente trabalho objetivou descrever a composição e a estrutura da comunidade de peixes que habita a praia arenosa da Ilha do Frade, Vitória, Espírito Santo, podendo, ainda servir como parâmetro para futuras comparações da qualidade ambiental da mesma bem como contribuir para o conhecimento das comunidades ícticas setentrionais da região sudeste.

\section{MATERIAL E MÉTODOS}

Área de estudo. A Ilha do Frade ( $\left.20^{\circ} 18^{\prime} \mathrm{S}, 40^{\circ} 17^{\prime} \mathrm{W}\right)$ está localizada próxima à entrada da Baia de Vitória (Fig. 1). O litoral da ilha é caracterizado por pequenas praias arenosas entre formações rochosas. A praia onde foram realizadas as amostragens possui $82 \mathrm{~m}$ de extensão e encontra-se na parte da ilha voltada para o continente. $\mathrm{O}$ limite sudeste é composto por costões rochosos enquanto a porção nordeste faz limite com a ponte que leva à Ilha de Vitória. A presença de grande biomassa de algas arribadas é constante ao longo do ano no local. 
Com base na classificação de CARTER (1988), a praia é considerada de domínio dissipativo devido ao baixo hidrodinamismo, apresentando ondas menores que $0,5 \mathrm{~m}$ de altura.

O local é freqüientemente utilizado por banhistas e está localizado em um dos bairros residenciais da cidade de Vitória. Pela falta de um sistema de tratamento de esgoto, a região do estudo sofre o aporte in natura de efluentes domésticos da ilha e de bairros adjacentes (Chagas et al., 2006).

Métodos de amostragem. As coletas foram realizadas mensalmente no período de maio/2004 a abril/ 2005 com uma rede de arrasto-de-praia (picaré) de $6 \mathrm{~m}$ de comprimento, $2 \mathrm{~m}$ de altura e malha com $10 \mathrm{~mm}$ de abertura entre nós. Em cada coleta foram realizados oito lances contíguos e paralelos à margem da praia, entre 6:00 e 12:00h, de acordo com os períodos de baixa-mar. Cada lance durou aproximadamente 5 minutos e percorreu uma área de 10 m de extensão.

Ao final de cada arrasto, os peixes capturados foram fixados em solução de formalina $10 \%$ e posteriormente conservados em álcool 70\%. No laboratório, os espécimes foram identificados de acordo com BOHLKE \& CHAPLIN (1968), Figueiredo \& Menezes (1978, 1980, 2000) e Menezes \& Figueiredo (1980, 1985). Os exemplares de cada espécie foram contados, pesados em balança semianalítica com precisão de $0,01 \mathrm{~g}$ e tiveram seus comprimentos totais $(\mathrm{mm})$ medidos. Juvenis e adultos foram classificados de acordo com o estádio de desenvolvimento gonadal, onde indivíduos com gônadas no estádio imaturo foram considerados juvenis e os demais adultos, seguindo-se a escala de VAZzOLER (1996). A partir destes dados foram calculadas a abundância relativa em número e biomassa e determinada a amplitude de

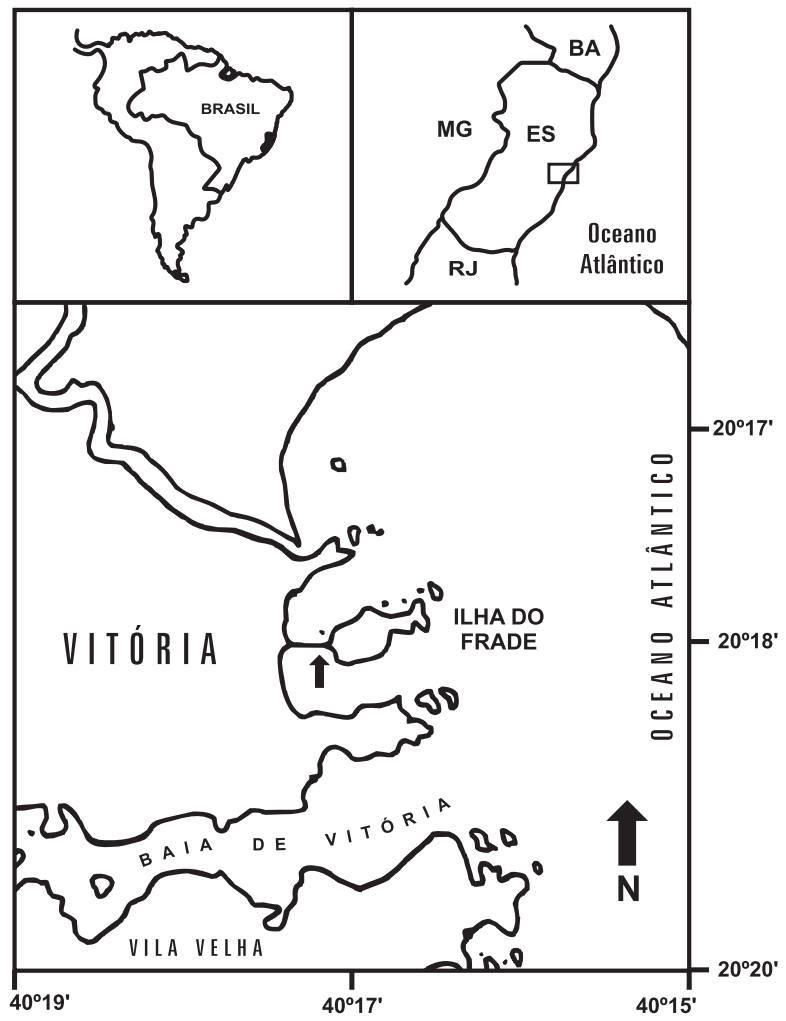

Fig. 1. Mapa da região da Ilha do Frade, Vitória, ES, indicando a praia arenosa amostrada. comprimento dos peixes capturados. Espécimes testemunhos foram depositados na coleção zoológica do Museu de Biologia Prof. Mello Leitão.

Análise dos dados. As médias mensais de biomassa e número de indivíduos foram testadas quanto à homogeneidade de variância (Teste de Levene) e normalidade das distribuições (Prova de KolmogorovSmirnov) de acordo com SoKal \& Rohlf (1981). Estas médias foram comparadas através de ANOVA, tendo como fator fixo o mês de coleta. Sempre que necessário, os dados foram transformados em $\log _{10}(\mathrm{x}+1)\left(\mathrm{ZAR}_{\mathrm{A}}, 1999\right)$. Quando os resultados indicaram diferenças significativas $(\mathrm{p}<0,05)$ foi aplicado o teste a posteriori de Newman-Keuls para averiguar quais meses diferiam entre si (ZAR, 1999).

Análise de agrupamento (Cluster, método normal) baseada na distância euclidiana foi utilizada para avaliar a similaridade na abundância entre os meses de coleta e na distribuição temporal das seis espécies mais representativas em número (aquelas que representaram mais de 5\% da captura total). A diversidade de espécies para cada mês foi calculada através do índice de diversidade de Shannon-Wiener (H'), como descrito em Pielou (1969).

\section{RESULTADOS}

Foram coletados 2.689 indivíduos compreendendo 13 ordens, 26 famílias e 45 espécies (Tab. I). Das espécies amostradas, $37(82,2 \%)$ foram representadas exclusivamente por exemplares juvenis e $8(17,8 \%)$ por juvenis e adultos. As famílias que apresentaram o maior número de espécies foram Sciaenidae (6), Carangidae (5), Lutjanidae (4) e Sparidae (3).

As amostragens mensais evidenciaram que as espécies mais importantes numericamente foram da família Lutjanidae (Lutjanus synagris (Linnaeus, 1758), L. jocu (Bloch \& Schneider, 1801), L. cyanopterus (Cuvler, 1828), e Ocyurus chrysurus (Bloch, 1791)) as quais contribuíram com $25,9 \%$. A esta família seguiram os Sparidae (Archosargus rhomboidalis (Linnaeus, 1758), A. probatocephalus (Walbaum, 1792) e Calamus penna (Valenciennes, 1830)) com 17,9\% bem como os Gerreidae (Eucinostomus lefroyi (Goode, 1874)) com 17,5\% da captura total na área. As espécies mais abundantes foram L. synagris (20,1\%), A. rhomboidalis $(17,7 \%)$, E. lefroyi $(17,5 \%)$ e Paralonchurus brasiliensis (Steindachner, 1875) (9,4\%). Dentre estas, apenas A. rhomboidalis esteve presente em todos os meses amostrados. Com relação ao peso, a espécie mais importante foi Cyclichthys spinosus (Linnaeus, 1758) com 42,3\% do peso total das capturas, seguida por A. rhomboidalis (13,5\%), E. lefroyi $(8,1 \%) \mathrm{e}$ L. synagris $(7,1 \%)$ (Tab. I).

A ictiofauna da praia arenosa da Ilha do Frade apresentou baixa dominância específica em relação ao número total de espécies, considerando que das 45 espécies registradas, 4 representaram $64,7 \%$ da captura total em número e as 41 espécies restantes representaram $35,3 \%$ do total.

Os maiores números de indivíduos foram registrados nos meses de março e dezembro, sendo os menores valores apresentados em junho e agosto (Fig. 2A). Os meses que apresentaram os maiores números de espécies foram dezembro e fevereiro, ao passo que janeiro e agosto apresentaram os menores números registrados (Fig. 2B). Os maiores valores de biomassa, entretanto, 
Tabela I. Valores específicos de abundância, abundância relativa (N\%), biomassa relativa (P\%) e amplitude do comprimento total (CT) das espécies de peixes amostradas mensalmente entre maio/2004 e abril/2005, na praia arenosa da Ilha do Frade, Vitória, ES.

\begin{tabular}{|c|c|c|c|c|c|c|c|c|c|c|c|c|c|c|c|}
\hline Famílias/ Espécies & Mai & Jun & Jul & Ago & Set & Out & Nov & Dez & Jan & Fev & Mar & Abr & $\mathrm{N} \%$ & $\mathrm{P} \%$ & $\mathrm{CT}(\mathrm{mm})$ \\
\hline LUTJANIDAE & & & & & & & & & & & & & & & \\
\hline $\begin{array}{l}\text { Lutjanus cyanopterus } \\
\text { (Cuvier, 1828) }\end{array}$ & 2 & 0 & 0 & 0 & 0 & 0 & 0 & 0 & 0 & 0 & 0 & 0 & 0,1 & 0,0 & $31-32$ \\
\hline $\begin{array}{l}\text { Lutjanus jocu } \\
\text { (Bloch \& Schnerider, 1801) }\end{array}$ & 30 & 31 & 12 & 17 & 10 & 0 & 0 & 2 & 6 & 8 & 1 & 13 & 4,8 & 5,1 & $20-110$ \\
\hline $\begin{array}{l}\text { Lutjanus synagris } \\
\text { (Linnaeus, 1758) }\end{array}$ & 23 & 0 & 20 & 7 & 8 & 17 & 4 & 16 & 16 & 102 & 268 & 59 & 20,1 & 7,1 & $21-333$ \\
\hline $\begin{array}{l}\text { Ocyurus chrysurus } \\
\text { (Bloch, 1791) } \\
\text { SCIAENIDAE }\end{array}$ & 0 & 0 & 0 & 0 & 0 & 4 & 0 & 3 & 0 & 2 & 14 & 2 & 0,9 & 0,2 & $25-56$ \\
\hline $\begin{array}{l}\text { Menticirrhus americanus } \\
\text { (Linnaeus, 1758) }\end{array}$ & 0 & 0 & 0 & 0 & 1 & 0 & 0 & 0 & 0 & 0 & 0 & 0 & 0 & 0,0 & $50-50$ \\
\hline $\begin{array}{l}\text { Umbrina coroides } \\
\text { Cuvier, } 1830\end{array}$ & 0 & 0 & 0 & 0 & 0 & 0 & 0 & 1 & 0 & 0 & 0 & 0 & 0 & 0,2 & $120-120$ \\
\hline $\begin{array}{l}\text { Paralonchurus brasiliensis } \\
\text { (Steindachner, 1875) }\end{array}$ & 0 & 0 & 0 & 0 & 131 & 6 & 114 & 2 & 0 & 0 & 0 & 0 & 9,4 & 1,2 & $22-71$ \\
\hline Stellifer rastrifer (Jordan, 1889) & 0 & 0 & 0 & 0 & 4 & 6 & 0 & 0 & 0 & 0 & 0 & 0 & 0,4 & 0,4 & $49-79$ \\
\hline Stellifer sp. & 1 & 0 & 3 & 0 & 0 & 0 & 0 & 0 & 0 & 0 & 0 & 0 & 0,1 & 1,5 & $110-150$ \\
\hline $\begin{array}{l}\text { Ophioscion punctatissimus } \\
\text { Meek \& Hildebrand, } 1925 \\
\text { CARANGIDAE }\end{array}$ & 0 & 0 & 0 & 0 & 12 & 21 & 10 & 8 & 0 & 7 & 0 & 0 & 2,2 & 1,8 & $27-97$ \\
\hline $\begin{array}{l}\text { Trachinotus falcatus } \\
\text { (Linnaeus, 1758) }\end{array}$ & 3 & 2 & 2 & 0 & 0 & 3 & 6 & 9 & 3 & 85 & 52 & 10 & 6,5 & 6,8 & $22-110$ \\
\hline $\begin{array}{l}\text { Trachinotus carolinus } \\
\text { (Linnaeus, 1766) }\end{array}$ & 0 & 0 & 0 & 0 & 0 & 0 & 0 & 0 & 0 & 0 & 1 & 0 & 0 & 0,0 & $55-55$ \\
\hline Selene vomer (Linnaeus, 1758) & 0 & 0 & 0 & 0 & 0 & 0 & 0 & 0 & 0 & 1 & 0 & 0 & 0 & 0,0 & $47-47$ \\
\hline $\begin{array}{l}\text { Oligoplites saurus } \\
\text { (Bloch \& Schnerider, 1801) }\end{array}$ & 1 & 0 & 0 & 0 & 0 & 0 & 0 & 0 & 0 & 24 & 0 & 0 & 0,9 & 0,2 & $26-57$ \\
\hline $\begin{array}{l}\text { Pseudocaranx dentex } \\
\text { (Bloch \& Schnerider, 1801) } \\
\text { SPARIDAE }\end{array}$ & 0 & 0 & 0 & 0 & 0 & 0 & 0 & 1 & 0 & 0 & 0 & 0 & 0 & 0,0 & $35-35$ \\
\hline Archosargus probatocephalus & 0 & 0 & 0 & 0 & 0 & 0 & 4 & 2 & 0 & 0 & 0 & 0 & 0,2 & 0,1 & $14-57$ \\
\hline $\begin{array}{l}\text { Archosargus rhomboidalis } \\
\text { (Linnaeus, 1758) }\end{array}$ & 51 & 25 & 56 & 47 & 78 & 15 & 18 & 33 & 36 & 21 & 93 & 4 & 17,7 & 13,6 & $16-109$ \\
\hline $\begin{array}{l}\text { Calamus penna } \\
\text { (Valenciennes, 1830) } \\
\text { GERREIDAE }\end{array}$ & 0 & 0 & 0 & 0 & 0 & 0 & 0 & 0 & 0 & 1 & 0 & 0 & 0 & 0,4 & $127-127$ \\
\hline $\begin{array}{l}\text { Eucinostomus lefroyi } \\
\text { (Goode, 1874) } \\
\text { SERRANIDAE }\end{array}$ & 19 & 23 & 1 & 0 & 15 & 0 & 117 & 110 & 59 & 54 & 42 & 30 & 17,5 & 8,1 & $13-110$ \\
\hline $\begin{array}{l}\text { Mycteroperca bonaci } \\
\text { (Poey, 1860) } \\
\text { HAEMULIDAE }\end{array}$ & 0 & 0 & 0 & 0 & 0 & 0 & 2 & 3 & 0 & 0 & 1 & 0 & 0,2 & 0,1 & $24-70$ \\
\hline $\begin{array}{l}\text { Haemulon steindachneri } \\
\text { (Jordan \& Gilbert, 1882) } \\
\text { EPHIPPIDAE }\end{array}$ & 1 & 1 & 0 & 0 & 0 & 0 & 1 & 0 & 0 & 0 & 1 & 1 & 0,2 & 0,5 & $42-108$ \\
\hline $\begin{array}{l}\text { Chaetodipterus faber } \\
\text { (Broussonet, 1782) } \\
\text { ALBULIDAE }\end{array}$ & 0 & 0 & 0 & 0 & 0 & 5 & 0 & 8 & 1 & 1 & 0 & 0 & 0,6 & 0,3 & $22-61$ \\
\hline $\begin{array}{l}\text { Albula vulpes } \\
\text { (Linnaeus, 1758) } \\
\text { CLUPEIDAE }\end{array}$ & 0 & 0 & 0 & 0 & 0 & 0 & 2 & 2 & 0 & 0 & 0 & 0 & 0,1 & 0,0 & $44-55$ \\
\hline $\begin{array}{l}\text { Harengula clupeola } \\
\text { (Cuvier, 1829) }\end{array}$ & 2 & 0 & 0 & 0 & 2 & 0 & 0 & 14 & 0 & 0 & 0 & 0 & 0,7 & 0,4 & $40-98$ \\
\hline $\begin{array}{l}\text { Jenkinsia lamprotaenia } \\
\text { (Gosse, 1851) } \\
\text { ENGRAULIDAE }\end{array}$ & 0 & 0 & 0 & 0 & 0 & 0 & 1 & 0 & 0 & 0 & 0 & 0 & 0 & 0,0 & $33-33$ \\
\hline $\begin{array}{l}\text { Anchovia clupeoides } \\
\text { (Swinson, 1839) } \\
\text { SYNGNATHIDAE }\end{array}$ & 0 & 6 & 0 & 14 & 7 & 14 & 20 & 61 & 0 & 1 & 0 & 0 & 4,6 & 0,6 & $14-77$ \\
\hline $\begin{array}{l}\text { Syngnathus scovelli } \\
\text { (Evermann \& Kendall, 1896) }\end{array}$ & 1 & 0 & 2 & 2 & 10 & 34 & 17 & 50 & 15 & 4 & 16 & 2 & 5,7 & 0,5 & $48-134$ \\
\hline $\begin{array}{l}\text { Hyppocampus reidi } \\
\text { Ginsburg, } 1933\end{array}$ & 0 & 0 & 0 & 0 & 0 & 0 & 0 & 0 & 0 & 0 & 0 & 1 & 0 & 0,1 & $110-110$ \\
\hline $\begin{array}{l}\text { SCORPAENIDAE } \\
\text { Scorpaena plumieri } \\
\text { (Bloch, 1797) } \\
\text { OSTRACIIDAE }\end{array}$ & 0 & 0 & 1 & 0 & 0 & 0 & 0 & 0 & 0 & 0 & 0 & 0 & 0 & 0,1 & $65-65$ \\
\hline $\begin{array}{l}\text { Acanthostracion sp. } \\
\text { HEMIRAMPHIDAE }\end{array}$ & 0 & 0 & 0 & 2 & 1 & 0 & 0 & 0 & 1 & 0 & 0 & 0 & 0,1 & 0,3 & $15-77$ \\
\hline $\begin{array}{l}\text { Hemiramphus brasiliensis } \\
\text { (Linnaeus, 1758) }\end{array}$ & 0 & 0 & 0 & 0 & 0 & 0 & 0 & 1 & 0 & 0 & 0 & 0 & 0 & 0,0 & $62-62$ \\
\hline
\end{tabular}




\begin{tabular}{|c|c|c|c|c|c|c|c|c|c|c|c|c|c|c|c|}
\hline abela I (cont.) & & & & & & & & & & & & & & & \\
\hline Famílias/ Espécies & Mai & Jun & Jul & Ago & Set & Out & Nov & Dez & Jan & Fev & Mar & Abr & $\mathrm{N} \%$ & P \% & $\mathrm{CT}(\mathrm{mm})$ \\
\hline $\begin{array}{l}\text { ATHERINOPSIDAE } \\
\text { Atherinella brasiliensis } \\
\text { (Quoy \& Gaimard, 1825) }\end{array}$ & 0 & 0 & 0 & 0 & 0 & 1 & 0 & 2 & 0 & 0 & 0 & 1 & 0,1 & 0,3 & $46-141$ \\
\hline OPHICHTHIDAE & & & & & & & & & & & & & & & \\
\hline $\begin{array}{l}\text { Myrophis punctatus } \\
\text { Lütken, } 1852\end{array}$ & 1 & 1 & 0 & 1 & 0 & 0 & 0 & 0 & 0 & 0 & 2 & 0 & 0,2 & 0,2 & $80-200$ \\
\hline PARALICHTHYIDAE & & & & & & & & & & & & & & & \\
\hline $\begin{array}{l}\text { Citharichthys arenaceus } \\
\text { Evermann \& Marsh, } 1902\end{array}$ & 0 & 0 & 0 & 0 & 0 & 0 & 0 & 1 & 0 & 0 & 0 & 0 & 0 & 0,1 & $118-118$ \\
\hline $\begin{array}{l}\text { Paralichthys brasiliensis } \\
\text { (Ranzani, 1842) } \\
\text { LABRISOMIDAE }\end{array}$ & 0 & 0 & 0 & 0 & 0 & 0 & 0 & 0 & 1 & 1 & 0 & 0 & 0,1 & 0,2 & $62-116$ \\
\hline $\begin{array}{l}\text { Labrisomus sp. } \\
\text { GOBIESOCIDAE }\end{array}$ & 0 & 1 & 0 & 0 & 0 & 0 & 0 & 0 & 0 & 0 & 0 & 0 & 0 & 0,0 & $50-50$ \\
\hline $\begin{array}{l}\text { Gobiesox strumosus } \\
\text { Cope, } 1870\end{array}$ & 0 & 0 & 0 & 0 & 1 & 0 & 0 & 0 & 0 & 0 & 0 & 0 & 0 & 0,0 & $41-41$ \\
\hline $\begin{array}{l}\text { POLYNEMIDAE } \\
\text { Polydactylus oligodon } \\
\text { (Gunther, 1860) } \\
\text { FISTULARIIDAE }\end{array}$ & 0 & 0 & 0 & 0 & 0 & 0 & 0 & 0 & 0 & 3 & 1 & 0 & 0,1 & 0,1 & $27-78$ \\
\hline $\begin{array}{l}\text { Fistularia petimba } \\
\text { Lacepède, } 1803 \\
\text { TRIGLIDAE }\end{array}$ & 0 & 0 & 2 & 0 & 0 & 0 & 0 & 0 & 1 & 0 & 1 & 0 & 0,1 & 0,2 & $144-370$ \\
\hline $\begin{array}{l}\text { Prionotus punctatus } \\
\text { (Bloch, 1793) } \\
\text { TETRAODONTIDAE }\end{array}$ & 0 & 0 & 0 & 0 & 0 & 0 & 0 & 1 & 0 & 0 & 0 & 0 & 0 & 0,1 & $70-70$ \\
\hline $\begin{array}{l}\text { Sphoeroides greeleyi } \\
\text { (Gilbert, 1900) }\end{array}$ & 1 & 1 & 3 & 5 & 2 & 2 & 3 & 0 & 0 & 7 & 6 & 2 & 1,2 & 2,8 & $25-126$ \\
\hline $\begin{array}{l}\text { Sphoeroides spengleri } \\
\text { Gilbert, } 1900\end{array}$ & 1 & 6 & 2 & 9 & 16 & 7 & 1 & 8 & 0 & 2 & 5 & 0 & 2,1 & 4,9 & $22-133$ \\
\hline $\begin{array}{l}\text { Sphoeroides testudineus } \\
\text { (Linnaeus, 1758) } \\
\text { DIODONTIDAE }\end{array}$ & 1 & 1 & 0 & 0 & 0 & 0 & 0 & 0 & 0 & 0 & 0 & 0 & 0,1 & 0,1 & $53-70$ \\
\hline $\begin{array}{l}\text { Cyclichthys spinosus } \\
\text { (Linnaeus, 1758) } \\
\text { MONACANTHIDAE }\end{array}$ & 0 & 8 & 18 & 10 & 13 & 1 & 3 & 4 & 0 & 0 & 0 & 0 & 2,1 & 42,3 & $42-148$ \\
\hline $\begin{array}{l}\text { Monacanthus ciliatus } \\
\text { (Mitchill, 1818) }\end{array}$ & 0 & 0 & 0 & 0 & 0 & 0 & 2 & 2 & 0 & 0 & 1 & 0 & 0,2 & 0,2 & $47-55$ \\
\hline
\end{tabular}

foram encontrados nos meses de setembro, julho e dezembro, sendo os menores em abril e janeiro (Fig. 2C). O número de exemplares capturados variou significativamente entre os meses (ANOVA: $\mathrm{F}_{11,84}=36,8$; $\mathrm{P}<0,01$ ), o mesmo ocorrendo com a biomassa (ANOVA: $\left.\mathrm{F}_{11,84}=59,0 ; \mathrm{P}<0,01\right)$. O teste a posteriori de NewmanKeuls evidenciou que março foi o mês que mais se diferenciou dos demais, em relação ao número médio de exemplares e em relação à biomassa foi julho.

$\mathrm{O}$ índice de diversidade de Shannon-Wiener $\left(\mathrm{H}^{\prime}\right)$ indicou pequenas variações entre os meses amostrados, sendo os maiores valores obtidos em outubro e dezembro e os menores em março e abril (Fig. 2D).

A análise de agrupamento utilizada para avaliar o nível de similaridade na abundância por mês de amostragem entre as seis espécies de Teleostei numericamente mais importantes desta praia evidenciou a existência de um grupo e uma espécie isolada (Fig. 3). O grupo foi constituído por A. rhomboidalis, L. synagris, Trachinotus falcatus (Linnaeus, 1758), S. scovelli (Evermann \& Kendall, 1896) e E. lefroyi, que estiveram presentes em, pelo menos, dez meses ao longo do período do estudo. Dentro do grupo, L. synagris e T. falcatus foram responsáveis pela maior similaridade, tendo suas abundâncias mais representativas nos meses correspondentes ao final do verão e início do outono. Paralonchurus brasiliensis (Ranzani, 1842) ficou isolada em decorrência de sua ocorrência restrita aos meses de primavera e início do verão.
A análise de agrupamento aplicada para avaliar a possível similaridade relacionando as abundâncias dos meses demonstrou a formação de três grupos (Fig. 4). O grupo I ficou dividido em três subgrupos, o primeiro formado pelos meses de maio e abril, o segundo por dezembro e janeiro e o terceiro por fevereiro e março. Todas as espécies selecionadas para esta análise, exceto P. brasiliensis, parecem ter contribuído para o aparecimento deste agrupamento, que resulta da ocorrência freqüente das mesmas em praticamente todas as coletas ao longo do período amostral e corresponde a períodos de grandes capturas em número de espécies e indivíduos. O grupo II ficou composto pelos meses de junho, julho, agosto e outubro. Este agrupamento reflete principalmente as maiores abundâncias de $A$. rhomboidalis, L. synagris e $S$. scovelli ocorridas entre julho e outubro, em relação às outras espécies analisadas. Os meses do inverno, os quais correspondem ao período das menores capturas em número de indivíduos com ocorrência de poucas espécies, foram alocados neste grupo. O grupo III foi constituído por setembro e novembro, representando o período de maior abundância de $P$. brasiliensis e E. lefroyi, ambas com pico de ocorrência sazonal nos meses de primavera. Neste período, ocorreram grandes capturas de indivíduos e de espécies. Foi revelada a existência de uma tendência sazonal para o local, além de alguma similaridade entre as estações do ano. Tal fato pode ser evidenciado pelo agrupamento 

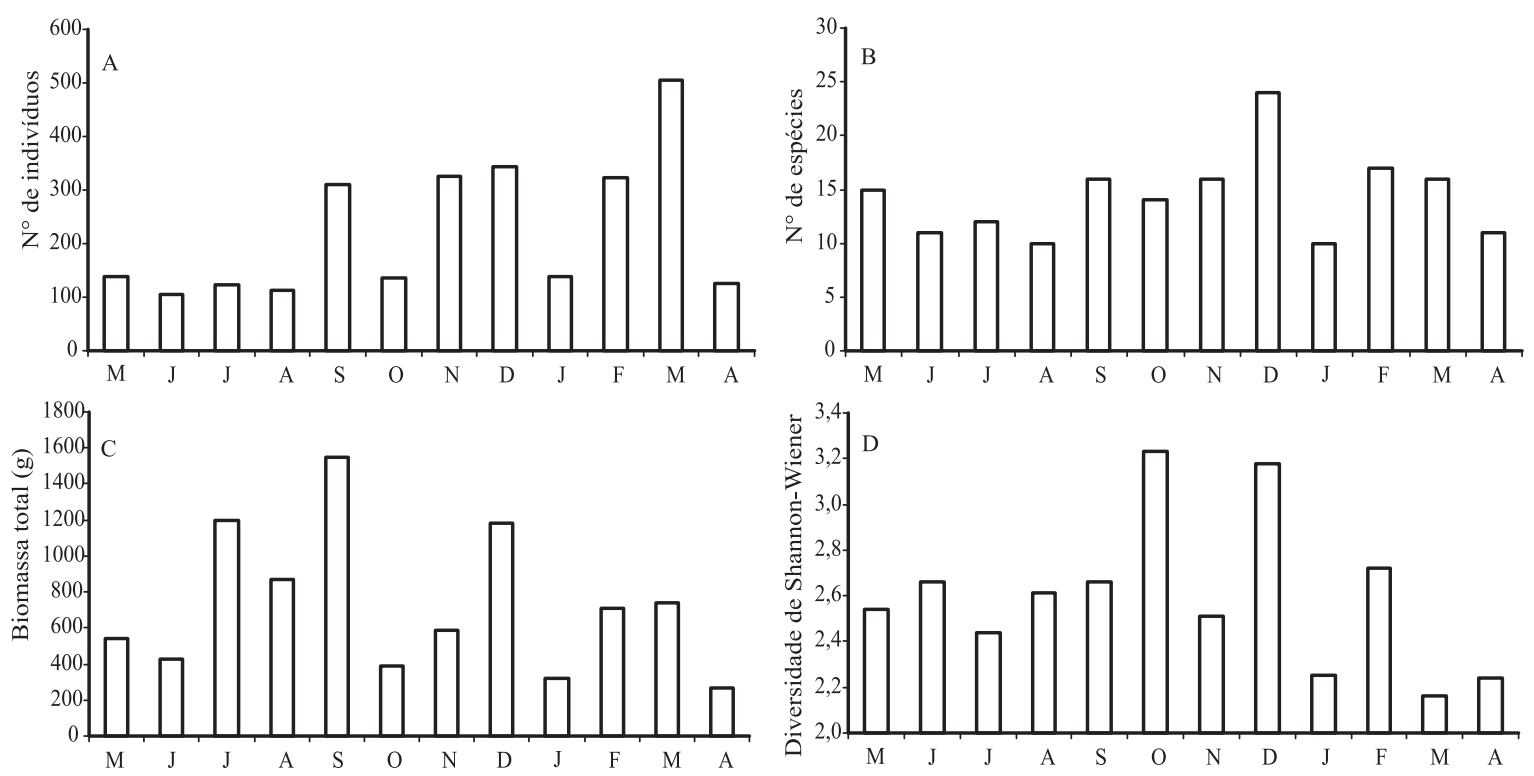

Fig. 2. Número de indivíduos (A), número de espécies (B), biomassa total (C) e diversidade de Shannon-Wiener (D) da comunidade de peixes amostrada mensalmente entre maio/2004 e abril/2005 na praia arenosa da Ilha do Frade, Vitória, ES.

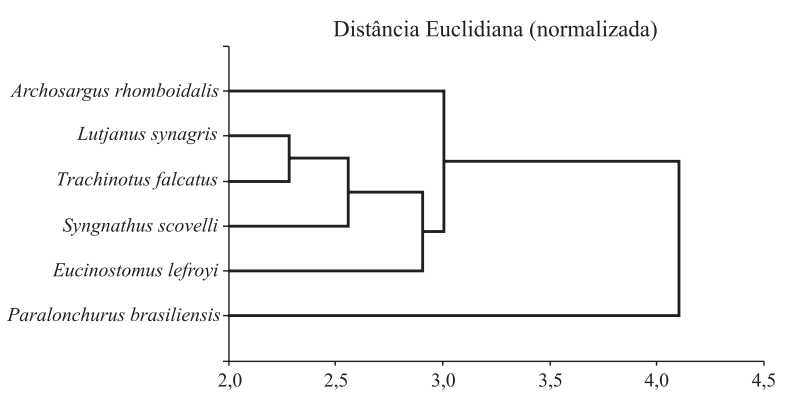

Fig. 3. Dendrograma da análise de agrupamento mostrando as similaridades na abundância entre as 6 espécies mais importantes amostradas mensalmente entre maio/2004 e abril/2005 na praia arenosa da Ilha do Frade, Vitória, ES.

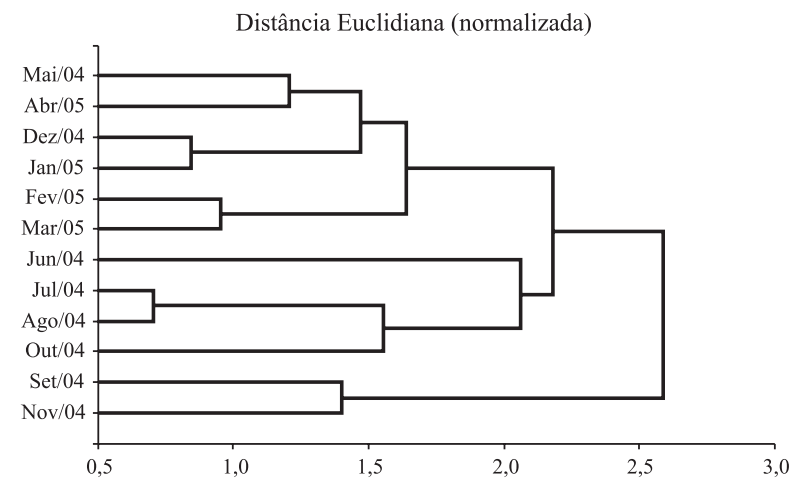

Fig. 4. Dendrograma da análise de agrupamento baseada nos dados de abundância das espécies mais representativas, mostrando as similaridades entre os doze meses de amostragens realizadas entre maio/2004 e abril/2005 na praia arenosa da Ilha do Frade, Vitória, ES.

dos meses de verão em um único grupo, assim como os do inverno. Os demais meses ficaram separados entre os grupos, demonstrando que o limite das estações não é nítido na ictiofauna local.

\section{DISCUSSÃO}

A comunidade de peixes que utiliza a praia arenosa da Ilha do Frade é composta principalmente por indivíduos jovens. Provavelmente por ser um local abrigado, com baixo hidrodinamismo e grande concentração de matéria orgânica, as espécies de peixes encontram condições favoráveis para alimentação e crescimento durante o início do seu ciclo de vida.

Assim como observado em outros estudos abordando a ictiofauna no litoral brasileiro (GODEFROID et al., 1997; TEIXEIRA \& ALMEIDA, 1998; GAELZER \& ZALMON, 2003 e GodEFroid et al., 2004), a assembléia estudada é dominada, em abundância e biomassa, por poucas espécies. Entretanto, uma quantidade relevante de espécies pode utilizar esses habitats esporadicamente como rota de migração, área de alimentação e reprodução (LASIAK, 1984).

Considerando a sua pequena extensão, a ictiofauna da praia da Ilha do Frade foi caracterizada por uma alta riqueza de espécies, se comparada a ictiofauna da PrainhaRJ (Gaelzer \& Zalmon, 2003), São Francisco do Itabapoana (Gomes et al., 2003) e de duas praias da Baía de Paranaguá-PR (Felix et al., 2006). Dois aspectos relevantes, nesse sentido, são o fato da praia estar próxima a formações rochosas submarinas e ser marcada durante todo o ano por um grande acúmulo de algas, das quais Ulva sp. (Ulvaceae) é dominante em todos os meses (obs. pess.). De acordo com Lowe-McConNELl (1987), o número de espécies das comunidades de peixes em águas tropicais está relacionado com a complexidade do ambiente. Esta relação se dá pela hipótese de que os peixes são atraídos para habitats estruturalmente heterogêneos devido à oferta de abrigos que dificultam a predação e a uma maior disponibilidade de alimento nesses locais (CARTER, 1988; DAY et al., 1989).

Dentre as espécies registradas, constatou-se a presença daquelas típicas de zonas de arrebentação (Trachinotus carolinus, T. falcatus, M. americanus e 
Pseudocaranx dentex) e de outras características de recifes coralinos ou rochosos (Monacanthus ciliatus, Archosargus probatocephalus, L. synagris, L. jocu e Scorpaena plumieri) (FIGUEIREDO \& MENEZES, 1980, 2000; Menezes \& Figueiredo, 1980).

$\mathrm{O}$ fato de o maior número de indivíduos ter sido observado nos meses de primavera e verão pode ser explicado pelo recrutamento de algumas espécies (e. g. E. lefroyi, L. synagris, A. clupeoides, S. scovelli) nesse período. Uma intensificação no número de espécies de peixes em atividade reprodutiva durante os meses mais quentes do ano tem sido amplamente descrita para o litoral brasileiro (GODEFROID et al., 1997; 2004; FELIX et al., 2006). Segundo vários autores, as maiores abundâncias de peixes em zonas costeiras das regiões tropicais e subtropicais do Atlântico Ocidental ocorrem no verão e as menores no inverno (GUNTHER, 1958; MCFARLAND, 1963; VENDEl et al., 2003). PesSANHA \& ARAúJo (2003) associaram o aumento no número de peixes à alta produtividade primária suportada pela descarga de nutrientes junto às águas continentais. O mesmo processo pode estar se repetindo na assembléia de peixes estudada em decorrência do aumento na pluviosidade durante o período de primavera e verão na região (INCAPER, 2007).

As diferenças significativas observadas nos valores médios de peso dos meses de julho e agosto em relação aos demais estão associadas às maiores abundâncias de $C$. spinosus, uma vez que essa espécie foi a mais representativa em biomassa. Os padrões encontrados na abundância mensal das espécies principalmente das mais representativas em número e peso podem estar relacionados a seus hábitos reprodutivos e subseqüente recrutamento à área estudada, visto que a maioria das espécies foram encontradas nos estágios iniciais do seu ciclo de vida.

O maior número de espécies encontrado no início dos meses de verão e de outono e o menor no inverno (Fig. 2B) são congruentes com os resultados de trabalhos realizados em duas praias arenosas da Baia de Sepetiba, Rio de Janeiro (Pessanha \& Araújo, 2003), na Ilha do Bom Abrigo, São Paulo (SAul \& CunNingham, 1995) e na zona de arrebentação de Pontal do Sul, Paraná (GODEFROID et al., 1997). Este resultado é explicado, principalmente, pela presença de juvenis de espécies com ocorrência esporádica, na região, durante essas estações.

A família Sciaenidae, registrada como a mais representativa em número de espécies no presente estudo, também foi considerada a mais importante em trabalhos realizados na região sudeste (RocHA \& RossiWongtschowski, 1998; Araúso et al., 1998) e sul (Muto et al., 2000; GODEFROID et al., 2004). Entretanto, 25,9\% do número total de indivíduos capturados pertenciam à família Lutjanidae, demonstrando a importância desse habitat para o desenvolvimento dessas espécies, que são comercialmente importantes na região.

A composição ictiofaunística da Ilha do Frade, comparada a de outras regiões do litoral brasileiro, teve maior semelhança com àquela da Baia de Sepetiba, Rio de Janeiro (PESSANHA \& ARAúso, 2003) e daquela presente no infralitoral raso do Balneário Atami, Paraná (GODEFROID et al., 2004), apresentando 18 espécies em comum com esses estudos. Trabalhos realizados na praia de Itapema, Bahia (Lopes et al., 1999) e na Ilha do Bom Abrigo, São Paulo (SAul \& CunNingham, 1995) apresentaram 15 espécies e em três praias arenosas do litoral de Maceió
(Teixeira \& Almeida, 1998), 11 espécies comuns ao presente estudo. Na Ilha do Frade-ES (Helmer \& Perrone, 1991) foram encontradas 148 espécies, das quais 31 também foram registradas nesse trabalho. Ao longo da costa do Brasil, a composição das comunidades de peixes difere entre as regiões norte e sul do país (FLOETER et al., 2001; FerReIRA et al., 2004; ChaGAS et al., 2006), sendo que o limite de distribuição da ictiofauna das duas regiões está entre os recifes de Abrolhos e o Cabo de São Tomé (CHAGAs et al., 2006). A localização geográfica da Ilha do Frade possibilita a ocorrência de espécies características das faunas tanto do sul como do norte, o que confere peculiaridade à ictiofauna da região. Vale ressaltar que as diferenças encontradas entre as áreas de estudos citadas, as variações interanuais ou até mesmo os petrechos de pesca empregados contribuem para a disparidade observada na composição das comunidades e na abundância relativa das espécies entre os distintos estudos (Rosa \& Odum, 1987).

O índice de Shannon-Wiener não revelou um padrão sazonal de diversidade para a assembléia de peixes da Ilha do Frade, mas mostrou que ocorre uma pequena oscilação na diversidade de espécies ao longo dos meses. Resultados semelhantes foram observados por SAUL \& Cunningham (1995) na Ilha do Bom Abrigo, São Paulo, onde apesar da ausência de um padrão sazonal de diversidade o verão foi significativamente diferente das outras três estações. LASIAK (1984), em uma praia da África do Sul, tampouco encontrou um padrão sazonal de diversidade na comunidade estudada. A ausência de uma sazonalidade bem definida nas regiões tropicais, com apenas duas estações (seca, inverno - com menor produtividade - e chuvosa, verão - com maior produtividade), provavelmente influencia na diversidade das comunidades de peixes destas latitudes, onde o verão tende a apresentar maiores valores de diversidade e quando existe maior similaridade entre as outras estações.

A dissimilaridade expressa pela análise de agrupamento (Cluster) na abundância mensal das seis espécies mais importantes numericamente e, principalmente, entre as três mais abundantes ( $L$. synagris, A. rhomboidalis e E. lefroyi) corrobora os resultados obtidos por GoDEFroID et al. (2004) na praia Balneário Atami, Paraná onde observaram-se padrões sazonais na distribuição das espécies dominantes em número (com alternações no pico de ocorrência das mesmas entre as estações). Essa distribuição temporal diferenciada das espécies dominantes parece ser uma estratégia adotada para minimizar a competição interespecífica e assim contribuir para a coexistência das mesmas no ecossistema.

Os resultados apresentados mostram a importância da área estudada para o desenvolvimento de juvenis de muitas espécies de peixes, inclusive de algumas economicamente importantes (e. g. L. jocu, L. synagris e $O$. chrysurus) e revelam que a preservação e correto manejo das mesmas são fundamentais para manutenção da biodiversidade e dos estoques pesqueiros locais. Todavia, são necessários mais estudos abordando, por exemplo, as relações tróficas para melhor compreender a forma como essa comunidade está estruturada.

Agradecimentos. Ao Dr. Rogério Luiz Teixeira pelo auxílio nos procedimentos estatísticos, aos Prof. M.Sc. Fabrício Saleme de Sá, M.Sc. Hélio Sá Santos e Dr. Marcelo Passamani 
pelos conhecimentos transmitidos e incentivo ao desenvolvimento da pesquisa e a dois revisores anônimos pela leitura crítica e sugestões enriquecedoras ao manuscrito.

\section{REFERÊNCIAS BIBLIOGRÁFICAS}

Araújo, F. G.; Cruz-Filho, A. G.; Azevedo, M. C. C. \& Santos, A. C. A. 1998. Estrutura da Comunidade de Peixes Demersais da Baía de Sepetiba, RJ. Revista Brasileira de Biologia $\mathbf{5 8}(3): 417-430$.

Bohlke, J. E. \& Chaplin, C. C. 1968. Fishes of the Bahamas and adjacent tropical waters. Wynnewood, Livingston Public Company. 771p.

Carter, R. W. G. 1988. Coastal Environments. An introduction to the physical ecological and cultural systems of coastlines. London, Academic. 617p.

Chagas, L. P.; Joyeux, J. C. \& Fonseca, F. R. 2006. Small-scale spatial changes in estuarine fish: subtidal assemblages in tropical Brazil. Journal of the Marine Biological Association of the United Kingdom 86:861-875.

Clark, B. M. 1997. Variation in surf-zone fish community structure across a wave expourse gradient. Estuarine, Coastal Shelf Science 44:659-674.

Clark, B. M.; Bennett, B. A. \& Lamberth, S. J. 1996. Factors affecting spatial variability in seine net catches of fish in the surf-zone of False Bay, South Africa. Marine Ecology Progress Series 131:17-34.

DAVIS, R. A., JR. 1985. Beach and nearshore zone. In: DAvis. R. A. $J_{R}$. ed. Coastal Sedimentary Environments. New York, Springer, Verlag. p.379-444.

Day, J. W., JR.; Hall, C. A. S.; Kemp, W. M. \& Yáñez-Arancibia, A. 1989. Estuarine Ecology. New York, Wiley-interscience. 543p.

Felix, F. C.; Spach, H. L.; Hackradt, C. W.; Moro, P. S. \& Rocha, D. C. 2006. Abundância sazonal e a composição da assembléia de peixes em duas praias estuarinas da Baía de Paranaguá, Paraná. Revista Brasileira de Zoociências 8:35-47.

Ferreira, C. E. L.; Floeter, S. R.; Gasparini, J. L.; Ferreira, B. P. \& Joyeux, J. C. 2004. Trophic struture patterns of Brazilian reef fishes: a latitudinal comparison. Journal of Biogeography 31:1093-1106.

Floeter, S. R.; Guimarães, R. Z. P.; Rocha, L. A.; Ferreira, C. E. L. Rangel, C. A. \& Gasparini, J. L. 2001. Biogeographic variation in reef-fish assemblages along the Brasilian coast. Global Ecology and Biogeography 10:423-433.

Figueiredo, J. L. \& Menezes, N. A. 1978. Manual de peixes marinhos do sudeste do Brasil. II. Teleostei (1). São Paulo, Museu de Zoologia USP. 110p.

1980. Manual de peixes marinhos do sudeste do Brasil. III. Teleostei (2). São Paulo, Museu de Zoologia USP. 90p

2000. Manual de peixes marinhos do sudeste do Brasil. VI. Teleostei (5). São Paulo, Museu de Zoologia USP. $116 \mathrm{p}$.

Gaelzer, R. L. \& Zalmon, I. R. 2003. The influence of wave gradient on the ichthyofauna of Southearn, Brazil: Focusing the community structure in surf zone. Journal of Coastal Research 35:456-462.

Godefroid, R. S.; HofstaetTer, M. \& Spach, H. L. 1997. Structure of the fish assemblage in the surf zone of the beach at Pontal do Sul, Paraná. Nerítica 11:77-93.

Godefroid, R. S.; Spach, H. L.; Santos, C.; Maclaren, G \& Schwarz JR., R. 2003. A fauna de peixes da praia do Balneário Atami, Paraná, Brasil. Atlântica 25(2):147-161.

2004. Mudanças temporais na abundância e diversidade da fauna de peixes do infralitoral raso de uma praia, sul do Brasil. Iheringia, Série Zoologia, 94(1):95-104.

Gomes, M. P.; Cunha, M. S. \& Zalmon, I. L. 2003. Spatial and temporal variations of diurnal ichthyofauna on surf-zone of São Francisco do Itabapoana beaches, Rio de Janeiro state, Brazil. Brazilian Archives of Biology and Technology 46(4):653-664

Gunther, G. 1958. Population studies of the shallow water fishes of an outer beach in south Texas. Publication Institute of Marine Sciences, University of Texas 5:186-193.
Helmer, J. L. \& Perrone, E. C. 1991. Composição preliminar da ictiofauna do litoral da Ilha do Frade, Vitória, ES. Revista cultural da UFES 44-45:69-78.

INCAPER. Instituto Capixaba de Pesquisa e Extensão RuRal. 2007. Estação Metereológica de Vitória, ES

Knoppers, B.; Ekau, W.; Figueiredo JR., E. A. G. \& Soares-Gomes, A 2002. Zona costeira e plataforma continental do Brasil. In Pereira, R. C. \& Soares-Gomes, A. Biologia Marinha. Rio de Janeiro, Interciência. p.353-360.

LAsiak, T. A. 1984. Structural aspects of the surf-zone fish assemblages at King's Beach, Algoa Bay, South Africa: longterm fluctuations. Estuarine, Coastal Shelf Science 18: 459-483

Lopes, P. R. D ; Silva, J. T. O.; Sena, M. P. Silva, I. S.; Veiga, D. C. M.; Silva, G. R. \& Santos, R. C. L. 1999. Contribuição ao conhecimento da ictiofauna da praia de Itapema, Santo Amaro da Purificação, Baia de Todos os Santos, Bahia. Acta Biológica Leopoldensia 21(1):99-105.

Lowe-McConnell, R. H. 1987. Ecological studies in tropical fish communities. New York, Cambrige University. 390p. McFarland, W. N. 1963. Seazonal change in the number and the biomass of fishes from the surf at Mustang Island, Texas. Publication Institute of Marine Science, University of Texas 9:91-112.

Menezes, N. A. \& Figueiredo, J. L. 1980. Manual de peixes marinhos do sudeste do Brasil. IV. Teleostei (3). São Paulo, Museu de Zoologia USP. 96p.

1985. Manual de peixes marinhos do sudeste do Brasil. V. Teleostei (4). São Paulo, Museu de Zoologia USP. $105 \mathrm{p}$.

Modde, T. 1980. Growth and residency of juvenile fishes within a surf-zone habitat in the Gulf of Mexico. Gulf Research Reports 4(6):377-385.

Muto, E. Y.; Soares, L. S. H. \& Rossi-Wongtschowski, C. L. D. B. 2000. Demersal fish assemblages of São Sebastião, Southeastern Brazil: structure and environmental conditioning factors (summer, 1994). Revista Brasileira de Oceanografia 48(1):2-9.

Pessanha, A. L. M. \& Araújo, F. G. 2003. Spatial, temporal and diel variations of fish assemblages at two sandy beaches in the Sepetiba Bay, Rio de Janeiro, Brazil. Estuarine, Costal and Shelf Science 57:817-828.

Pileou, E. C. 1969. The measurement of diversity in different types of biological colections. Journal of Theoretical Biology 13:131-144

Rocha, G. R. A. \& Rossi-Wongtschowski, C. L. D. B. 1998 Demersal fish comunnity on the inner shelf of Ubatuba, Southeastern Brazil. Revista Brasileira de Oceanografia 46(2):93-109.

Rosa, L. P. \& Odum, W. E. 1987. Use of tidal freshwater mashes by fishes and macrofaunal crustaceans along a marsh streamorder gradient. Estuaries 10(1):36-43.

Ruple, D. L. 1984. Occurrence of larval fishes in the surf zone of a Northern Gulf of México Barrier Islad. Estuarine, Coasta Shelf Science 18:191-208.

Saul, A. C. \& Cunningham, P. T. M. 1995. Comunidade ictiofaunística da Ilha do Bom Abrigo, Cananéia, São Paulo, Brasil, 2-lanço. Arquivo de Biologia e Tecnologia 38(4): 1053-1069.

SoKal, R. R. \& Rohlf, F. J. 1981. Biometry. New York, W. H. Freeman. 859p.

Teixeira, R. L.F FAlCão, G. A. F. \& Melo, S. C. 1992. Ocorrência e alimentação de Sciaenidae (Pisces: Perciformes) nas zonas de arrebentação de praias de Maceió, Brasil. Atlântica 14:29-42.

Teixeira, R. L. \& Almeida, G. L. 1998. Composição da ictiofauna de três praias arenosas de Maceió, AL. Boletim do Museu de Biologia Mello Leitão 15(3):34-37.

VAZZOLER, A. E. M. 1996. Biologia da reprodução de peixes teleósteos: teoria e prática. Maringá, EDUEM. 169p.

Vendel, A. L.; Lopes, S. G.; Santos, C. \& Spach, H. L. 2003. Fish Assemblages in a Tidal Flat. Brazilian Archives of Biology and Technology 46(2):233-242.

ZAR, J. H. 1999. Biostatistical Analysis. New Jersey, PrenticeHall. $718 \mathrm{p}$. 